PROCEEDINGS OF THE

AMERICAN MATHEMATICAL SOCIETY

Volume 138, Number 6, June 2010, Pages 2141-2150

S 0002-9939(10)10264-0

Article electronically published on February 9, 2010

\title{
THE SPECTRAL SHIFT FUNCTION FOR COMPACTLY SUPPORTED PERTURBATIONS OF SCHRÖDINGER OPERATORS ON LARGE BOUNDED DOMAINS
}

\author{
PETER D. HISLOP AND PETER MÜLLER
}

(Communicated by Walter Craig)

\begin{abstract}
We study the asymptotic behavior as $L \rightarrow \infty$ of the finite-volume spectral shift function for a positive, compactly supported perturbation of a Schrödinger operator in $d$-dimensional Euclidean space, restricted to a cube of side length $L$ with Dirichlet boundary conditions. The size of the support of the perturbation is fixed and independent of $L$. We prove that the Cesàro mean of finite-volume spectral shift functions remains pointwise bounded along certain sequences $L_{n} \rightarrow \infty$ for Lebesgue-almost every energy. In deriving this result, we give a short proof of the vague convergence of the finite-volume spectral shift functions to the infinite-volume spectral shift function as $L \rightarrow \infty$. Our findings complement earlier results of W. Kirsch [Proc. Amer. Math. Soc. 101, 509-512 (1987); Int. Eqns. Op. Th. 12, 383-391 (1989)], who gave examples of positive, compactly supported perturbations of finite-volume Dirichlet Laplacians for which the pointwise limit of the spectral shift function does not exist for any given positive energy. Our methods also provide a new proof of the BirmanSolomyak formula for the spectral shift function that may be used to express the measure given by the infinite-volume spectral shift function directly in terms of the potential.
\end{abstract}

\section{Statement of The PROBlem AND RESUlt}

The spectral shift function (SSF) plays an important role in scattering theory for Schrödinger operators $[\mathrm{Y}$. For the particular case of a quantum mechanical system in a finite volume, the SSF, as a function of energy $E$, counts the change in the number of eigenvalues below $E$ due to adding a perturbing potential $V$.

We are interested in the following question: given cubes $\Lambda_{L} \subset \mathbb{R}^{d}$ in $d$-dimensional Euclidean space, which are centered at the origin and have side lengths $L>0$, what is the limiting behavior as $L \rightarrow \infty$ of the SSF corresponding to the Laplacian plus a background potential $H_{0}^{(L)}:=-\left(\Delta_{L} / 2\right)+V_{0}$ and its perturbation $H_{1}^{(L)}:=H_{0}^{(L)}+V$ ? Both operators are defined on the Hilbert space $L^{2}\left(\Lambda_{L}\right)$ with Dirichlet boundary conditions. The potentials $V_{0}$ and $V$ act as multiplication operators corresponding to real-valued functions $V_{0}$ and $V$ such that

$$
\begin{aligned}
& \max \left\{0, V_{0}\right\} \in \mathcal{K}_{\text {loc }}\left(\mathbb{R}^{d}\right), \quad \max \left\{0,-V_{0}\right\} \in \mathcal{K}\left(\mathbb{R}^{d}\right), \\
& V \in \mathcal{K}_{\text {loc }}\left(\mathbb{R}^{d}\right), \quad V \geqslant 0, \quad \operatorname{supp}(V) \text { is compact. }
\end{aligned}
$$

Received by the editors September 3, 2009.

2010 Mathematics Subject Classification. Primary 81U05, 35P15, 47A40; Secondary 47A75.

The first author was supported in part by NSF grant 0503784 while this work was being done. 
It is understood in (因) that the compact support of $V$ is independent of $L$, and we have written $\mathcal{K}\left(\mathbb{R}^{d}\right)$ and $\mathcal{K}_{\text {loc }}\left(\mathbb{R}^{d}\right)$ to denote the Kato class and the local Kato class, respectively $[\mathrm{AS}$, $\mathrm{S} 2$. We also introduce the corresponding infinite-volume self-adjoint Schrödinger operators $H_{0}:=-(\Delta / 2)+V_{0}$ and $H_{1}:=H_{0}+V$ on $\mathrm{L}^{2}\left(\mathbb{R}^{d}\right)$.

The self-adjoint operators $H_{0}^{(L)}$ and $H_{1}^{(L)}$ have compact resolvents and, therefore, discrete spectrum. For a given energy $E \in \mathbb{R}$, let $N_{0}^{(L)}(E)$, resp. $N_{1}^{(L)}(E)$, denote the number of eigenvalues, including multiplicity, for $H_{0}^{(L)}$, resp. $H_{1}^{(L)}$, less than or equal to $E$. These are both monotone increasing functions of the energy $E$. We define the relative eigenvalue counting function by

$$
E \mapsto \xi_{L}(E) \equiv \xi\left(E ; H_{1}^{(L)}, H_{0}^{(L)}\right):=N_{0}^{(L)}(E)-N_{1}^{(L)}(E) \geqslant 0
$$

for all $E \in \mathbb{R}$. It is known that this function is equal to the (more generally defined) spectral shift function for the pair $\left(H_{1}^{(L)}, H_{0}^{(L)}\right)$; see e.g. [Y, BiY] or (5.1) in the Appendix. A basic question is the pointwise boundedness with respect to the energy of the SSF $\xi_{L}$ as $L \rightarrow \infty$.

The main result of this paper is Theorem 1.3, which states that the Cesàro mean of subsequences of $\xi_{L}$ is bounded from above by the infinite-volume SSF $\xi$ Lebesguealmost everywhere. Here, the infinite-volume SSF $\xi$ for the pair $\left(H_{1}, H_{0}\right)$ is defined in terms of the invariance principle and Krĕn's trace identity; see Remark 5.1 in the Appendix. Theorem 1.3 relies on an abtract result of Komlós $[\mathrm{Ko}$ and vague convergence of the measures $\xi_{L}(E) \mathrm{d} E$ to the measure $\xi(E) \mathrm{d} E$, where $\mathrm{d} E$ denotes the Lebesgue measure on $\mathbb{R}$. We include a short proof of the vague convergence of the finite-volume spectral shift measures. As a by-product of our analysis we obtain a short proof of the Birman-Solomyak formula for the SSF of Schrödinger operators that seems to be new.

The main motivation of this paper comes from two papers of W. Kirsch [K1, K2, who considered the (un-)boundedness of the $\operatorname{SSF} \xi_{L}$ as $L \rightarrow \infty$. Weyl's law indicates that the leading behavior of each eigenvalue counting function in (1.1) is the same and proportional to the volume $L^{d}$. Since the support of $V$ is compact and independent of $L$, one might think that all $L$-dependence in (1.1) cancels out and that the SSF remains bounded as $L \rightarrow \infty$. For the corresponding discrete problem in $\ell^{2}\left(\mathbb{Z}^{d}\right)$, this is indeed true as can be seen from a finite-rank perturbation argument. For the continuum problem, however, which we consider here, Kirsch showed that this intuition is wrong in dimensions $d \geqslant 2$ if $V_{0} \equiv 0$. (In $d=1$ the finite-rank perturbation argument is also applicable in the continuum.)

Theorem 1.1 ([K1]). Let $d \in \mathbb{N} \backslash\{1\}$ and assume in addition to (因) that $V_{0}=0$ and $V \in \mathrm{L}^{\infty}\left(\mathbb{R}^{d}\right)$. Then, for any $E>0$, we have

$$
\sup _{L>0} \xi_{L}(E)=\infty \text {. }
$$

Furthermore, there is a countable, dense set of energies $\mathcal{E} \subset[0, \infty[$ so that for any $E \in \mathcal{E}$, we have

$$
\sup _{L \in \mathbb{N}} \xi_{L}(E)=\infty
$$

The same is true for perturbations by boundary conditions: as a corollary of Theorem 1.1, Kirsch [K1] considered the Dirichlet Laplacian on $\Lambda_{L}$ with an additional Dirichlet wall along the boundary $\partial \Lambda_{\ell}$ of an arbitrary fixed cube $\Lambda_{\ell} \subset \Lambda_{L}$. He compared the eigenvalue counting function $N_{D, \ell}^{(L)}$ for this operator, which is a 
direct sum of two Dirichlet Laplacians, to the one obtained by placing Neumann boundary conditions along $\partial \Lambda_{\ell}$. He concluded that $N_{N, \ell}^{(L)}(E)-N_{D, \ell}^{(L)}(E)$ has an infinite supremum over $L$, in the same way as in Theorem 1.1. We remark that, similarly, this effect also shows up when comparing $N_{D, \ell}^{(L)}$ to the eigenvalue counting function of the Dirichlet Laplacian on $\Lambda_{L}$.

In the general case where $V_{0}$ is not identically zero, much less is known. In fact, Kirsch's proof [K1] of Theorem 1.1] uses the high degeneracy of the eigenvalues of $-\Delta_{L}$ to deduce the claimed divergence of the SSF. In general, the perturbation $V_{0}$ removes this degeneracy.

In [K2, Kirsch aimed at a complementary statement to Theorem 1.1] asking for finiteness of the SSF for energies outside the bad countable set in (1.3). The result he got, however, requires $V$ to become smaller and smaller in norm when $L$ tends to infinity.

Theorem $1.2(\mathrm{~K} 2)$. Let $d \in \mathbb{N}$ and assume in addition to (因) that $V_{0}, V \in$ $\mathrm{L}^{\infty}\left(\mathbb{R}^{d}\right)$. Define $V_{L}:=L^{-k} V$ for some arbitrary $k>d+1$ and $\bar{H}_{1}^{(L)}:=H_{0}^{(L)}+V_{L}$. Then, there is a set $S \subset \mathbb{R}$ of full Lebesgue measure such that for every $E \in S$ we have

$$
\lim _{L \rightarrow \infty} \bar{\xi}_{L}(E)=0
$$

for the $S S F \bar{\xi}_{L}$ of the pair $\left(\bar{H}_{1}^{(L)}, H_{0}^{(L)}\right)$.

In our proof of a lower bound on the density of states for random Schrödinger operators $[\mathrm{HM}]$, we were also led to consider the question of the boundedness of the finite-volume SSF. In the following theorem we prove an almost everywhere upper bound on the Cesàro mean of subsequences of $\xi_{L}$. Our result naturally complements Kirsch's Theorem 1.1, which is commonly cited as an example of a pathological behavior of the SSF.

Theorem 1.3. Let $d \in \mathbb{N}$ and assume (因). Then, for every sequence of lengths $\left.\left(L_{j}\right)_{j \in \mathbb{N}} \subset\right] 0, \infty\left[\right.$ with $\lim _{j \rightarrow \infty} L_{j}=\infty$, there exists a subsequence $\left(j_{i}\right)_{i \in \mathbb{N}} \subset \mathbb{N}$ with $\lim _{i \rightarrow \infty} j_{i}=\infty$ such that for every subsequence $\left(i_{k}\right)_{k \in \mathbb{N}} \subset \mathbb{N}$ with $\lim _{k \rightarrow \infty} i_{k}=\infty$ we have

$$
\lim _{K \rightarrow \infty} \frac{1}{K} \sum_{k=1}^{K} \xi_{\widetilde{L}_{k}}(E) \leqslant \xi(E)
$$

for Lebesgue-almost all $E \in \mathbb{R}$. Here we have set $\widetilde{L}_{k}:=L_{j_{i_{k}}}$ for all $k \in \mathbb{N}$.

The assumption that $V$ has compact support could be relaxed. There have been many works on the boundedness of finite-volume spectral shift functions for Schrödinger operators. Hundertmark, Killip, Nakamura, Stollmann and Veselić HuKNSV] have obtained a bound on $\int_{\mathbb{R}} \mathrm{d} E \xi_{L}(E) f(E)$ for bounded, compactly supported functions $f$. Combes, Hislop and Nakamura CHN proved an $\mathrm{L}^{p}$-bound on the SSF for pairs of operators $(A, B)$ for which $C=B-A$ is in the Schattenvon Neumann trace ideal $\mathcal{I}_{1 / p}$, with $1 \leqslant p<\infty$. This was improved by Hundertmark and Simon [HuS], who obtained an optimal bound on the $L^{p}$-norm of the SSF. Sobolev [So, Sect. 4] showed continuity of the infinite-volume SSF $\xi$ for pairs of Schrödinger operators with $V_{0}=0$ and proved a pointwise bound on $\xi(E)$ for sufficiently large energies (there are more general results in [So, Sect. 4] in an abstract 
setting). For the case of random Schrödinger operators on $L^{2}\left(\mathbb{R}^{d}\right)$, it is known that the expectation of the finite-volume SSF is pointwise bounded CHK1, CHK2.

The proof of Theorem 1.3 is deferred to Section 3. It relies on a deep result of Komlós [Ko, Thm. 1a] for $\mathrm{L}^{1}$-bounded sequences. We infer this condition from the non-negativity of $\xi_{L}$ and from vague convergence of the finite-volume SSF towards the infinite-volume SSF. The latter is the content of

Theorem 1.4. Let $d \in \mathbb{N}$ and assume (因). Then, we have

$$
\lim _{L \rightarrow \infty} \int_{\mathbb{R}} \mathrm{d} E \xi_{L}(E) f(E)=\int_{\mathbb{R}} \mathrm{d} E \xi(E) f(E)
$$

for every continuous and compactly supported function $f \in C_{c}(\mathbb{R})$ and for every indicator function $f=\chi_{I}$ of some interval $I \subset \mathbb{R}$. In particular, for Lebesguealmost all $E \in \mathbb{R}$ we have

$$
\lim _{\delta \downarrow 0} \lim _{L \rightarrow \infty} \frac{1}{\delta} \int_{E}^{E+\delta} \mathrm{d} E^{\prime} \xi_{L}\left(E^{\prime}\right)=\xi(E) .
$$

Remarks 1.5. (i) Geisler, Kostrykin, and Schrader [GKS, Thm. 3.3] proved that the distribution functions of the finite-volume spectral shift measures $\xi_{L}(E) \mathrm{d} E$ converge to the distribution function of the infinite-volume spectral shift measure, which is equivalent to vague convergence for the measures under consideration. Their proof (explicitly given for the case $d=3$, but the argument generalizes) applies to real-valued measurable potentials $V \in \ell^{1}\left(\mathrm{~L}^{2}\right)$, the Birman-Solomyak space. The proof also requires a Weyl asymptotic of the SSF for high energies, which is not necessary however.

(ii) Kirsch's result (1.2) shows that one cannot get rid of the energy smoothing in (1.7); that is, the limits $\delta \downarrow 0$ and $L \rightarrow \infty$ must not be interchanged. The best one could hope for is convergence Lebesgue-almost everywhere of $\left(\xi_{L_{j}}\right)_{j \in \mathbb{N}}$ for sequences of diverging lengths. Theorem 1.3 is a partial result in this direction based.

(iii) For the sake of concreteness, we mention an example of a toy family $\left(\zeta_{L}\right)_{L>0}$ of functions

$$
\mathbb{R} \ni E \mapsto \zeta_{L}(E):=\left\{\begin{array}{cc}
L, & E \in\{\mathbb{Q}+[L]\} \\
0, & \text { otherwise }
\end{array}\right.
$$

which captures the properties that are known for the family of spectral shift functions $\left(\xi_{L}\right)_{L>0}$. In (1.8) we have written $[L]:=L \bmod 1$ for the fractional part of $L$ in $\left[0,1\left[\right.\right.$. Indeed, $\lim _{L \rightarrow \infty} \zeta_{L}(E)$ does not exist for any $E \in \mathbb{R}$, and the corresponding suprema over $L$ are infinite as in (1.2) and (1.3). The limits (1.6), (1.7) are zero for the toy family. Note that, in addition, the sequence of functions $\left(\zeta_{L_{j}}\right)_{j \in \mathbb{N}}$ converges to zero Lebesgue-almost everywhere for every diverging sequence $\left(L_{j}\right)_{j \in \mathbb{N}}$ of lengths.

The Birman-Solomyak formula BiS, $\mathrm{S3}$, is an important identity relating the SSF to the perturbation potential $V$. We state it in the next theorem and give a short proof of it in Section 4, valid under our assumptions (因). Even though our proof of Theorem 1.4 does not rely upon the Birman-Solomyak formula, they are both related in spirit and are based on the Feynman-Kac formula. We write $\chi_{B}$ for the indicator function of the set $B \subset \mathbb{R}$.

Theorem 1.6. Let $H_{0}$ and $H_{1}$ be as above with potentials $V_{0}$ and $V$ satisfying (因), and let $H_{\lambda}:=H_{0}+\lambda V$ on $\mathrm{L}^{2}\left(\mathbb{R}^{d}\right)$ for $\lambda \in[0,1]$. Then the (infinite-volume) SSF $\xi$ 
for the pair $\left(H_{1}, H_{0}\right)$ satisfies the Birman-Solomyak formula

$$
\int_{B} \mathrm{~d} E \xi(E)=\int_{0}^{1} \mathrm{~d} \lambda \operatorname{tr}\left[V^{1 / 2} \chi_{B}\left(H_{\lambda}\right) V^{1 / 2}\right]
$$

for every Borel set $B \subset \mathbb{R}$.

Remarks 1.7. (i) We allow both sides of (1.9) to be $+\infty$. If $\sup B<\infty$, then

$$
\operatorname{tr}\left[V^{1 / 2} \chi_{B}\left(H_{\lambda}\right) V^{1 / 2}\right]=\int_{\mathbb{R}^{d}} \mathrm{~d} x V(x) \chi_{B}\left(H_{\lambda}\right)(x, x)<\infty
$$

by $[\mathrm{Br}$, Cor. 4.4], the continuity of the integral kernel of the spectral projection (see [AS, Prop. 4.3] or [S2, Thm. B.7.1(d)]) and since $V \in \mathcal{K}\left(\mathbb{R}^{d}\right) \subseteq \mathrm{L}_{\text {unif, loc }}^{1}\left(\mathbb{R}^{d}\right)$ has compact support. Moreover, this trace is uniformly bounded in $\lambda \in[0,1]$.

(ii) The Birman-Solomyak formula provides another representation of the limiting measure $\xi(E) \mathrm{d} E$ in Theorem 1.4, Using (1.9), Equation (1.6) reads

$$
\lim _{L \rightarrow \infty} \int_{\mathbb{R}} \mathrm{d} E \xi_{L}(E) f(E)=\int_{0}^{1} \mathrm{~d} \lambda \operatorname{tr}\left[V^{1 / 2} f\left(H_{\lambda}\right) V^{1 / 2}\right] .
$$

(iii) Simon remarks that his more general Birman-Solomyak formula [S3, Thm. 4] includes the case of Schrödinger operators with slightly different conditions on the potentials than ours. For example, one may take $V_{0}$ and $V$ to be uniformly Kato class and $V \geqslant 0$ in the class $\ell_{1}\left(L^{2}\right)$.

\section{Proof of vague convergence, Theorem 1.4}

In this section we prove vague convergence of the finite-volume SSF in the macroscopic limit. In contrast to GKS] we do not use the Weyl asymptotics of the SSF for high energies.

Let $L, t>0$. The standard Feynman-Kac representation [S1] of the heat kernel gives

$$
\begin{aligned}
\widetilde{\xi}_{L}(t) & :=\int_{\mathbb{R}} \mathrm{d} E \mathrm{e}^{-t E} \xi_{L}(E) \\
& =\frac{1}{t} \frac{1}{(2 \pi t)^{d / 2}} \int_{\Lambda_{L}} \mathrm{~d} x \mathbb{E}_{x, x}^{0, t}\left[\chi_{\Lambda_{L}}^{t}(b) \mathrm{e}^{-\int_{0}^{t} \mathrm{~d} s V_{0}(b(s))}\left(1-\mathrm{e}^{-\int_{0}^{t} \mathrm{~d} s V(b(s))}\right)\right] .
\end{aligned}
$$

Here, $\mathbb{E}_{x, y}^{0, t}$ denotes the normalized expectation over all Brownian bridge paths $b$ starting at $x \in \mathbb{R}^{d}$ at time $s=0$ and ending at $y \in \mathbb{R}^{d}$ at time $s=t$. The Dirichlet boundary condition is taken into account by the cut-off functional $\chi_{\Lambda_{L}}^{t}(b)$, which is equal to one if $b(s) \in \Lambda_{L}$ for all $s \in[0, t]$, and zero otherwise.

First, we rewrite the term in parentheses in (2.1) as an integral

$$
1-\mathrm{e}^{-\int_{0}^{t} \mathrm{~d} s V(b(s))}=\int_{0}^{1} \mathrm{~d} \lambda\left(\int_{0}^{t} \mathrm{~d} s^{\prime} V\left(b\left(s^{\prime}\right)\right)\right) e^{-\lambda \int_{0}^{t} \mathrm{~d} s V(b(s))}
$$

over a parameter $\lambda \in[0,1]$. Then, we translate the Brownian paths $b(s)$ to $b(s)+x$ and use Fubini's Theorem so that

$$
\widetilde{\xi}_{L}(t)=\frac{1}{t} \frac{1}{(2 \pi t)^{d / 2}} \int_{0}^{1} \mathrm{~d} \lambda \mathbb{E}_{0,0}^{0, t}\left[F_{L}(\lambda, t ; b)\right]
$$

where

$$
F_{L}(\lambda, t ; b):=\int_{\Lambda_{L}} \mathrm{~d} x \chi_{\Lambda_{L}}^{t}(b+x)\left(\int_{0}^{t} \mathrm{~d} s^{\prime} V\left(b\left(s^{\prime}\right)+x\right)\right) \mathrm{e}^{-\int_{0}^{t} \mathrm{~d} s U_{\lambda}(b(s)+x)}
$$


and $U_{\lambda}:=V_{0}+\lambda V$. Clearly, $F_{L}(\lambda, t ; b) \geqslant 0$ is monotone increasing in $L$ for every Brownian bridge path $b$, every $\lambda \in[0,1]$ and every $t>0$. Therefore, the Monotone Convergence Theorem gives the pointwise limit

$$
\lim _{L \rightarrow \infty} \widetilde{\xi}_{L}(t)=\frac{1}{(2 \pi t)^{d / 2}} \int_{0}^{1} \mathrm{~d} \lambda \int_{\mathbb{R}^{d}} \mathrm{~d} x \int_{0}^{t} \frac{\mathrm{d} s}{t} \mathbb{E}_{x, x}^{0, t}\left[V(b(s)) \mathcal{U}_{t}(b)\right]
$$

for all $t>0$, where we have introduced the Brownian functional

$$
\mathcal{U}_{t}(b):=\exp \left\{-\int_{0}^{t} \mathrm{~d} s U_{\lambda}(b(s))\right\}
$$

and used Fubini's Theorem. We will see shortly that the limit (2.5) is finite, which, a posteriori, will justify the final interchange of integrations (for a remark on boundedness in $L$ see [KosS, Ex. 4.2]).

Now we show that the limit (2.5) is equal to the two-sided Laplace transform of the infinite-volume SSF. It is well-defined for $t>0$ (see Remark [5.1) and given by

$$
\begin{aligned}
\widetilde{\xi}(t) & :=\int_{\mathbb{R}} \mathrm{d} E \mathrm{e}^{-t E} \xi\left(E ; H_{1}, H_{0}\right)=-\int_{\mathbb{R}} \mathrm{d} E \mathrm{e}^{-t E} \xi\left(\mathrm{e}^{-t E} ; \mathrm{e}^{-t H_{1}}, \mathrm{e}^{-t H_{0}}\right) \\
& =-\frac{1}{t} \int_{0}^{\infty} \mathrm{d} \eta \xi\left(\eta ; \mathrm{e}^{-t H_{1}}, \mathrm{e}^{-t H_{0}}\right)=\frac{1}{t} \int_{\mathbb{R}} \mathrm{d} \eta \xi\left(\eta ; \mathrm{e}^{-t H_{0}}, \mathrm{e}^{-t H_{1}}\right) \\
& =\frac{1}{t} \operatorname{tr}\left(\mathrm{e}^{-t H_{0}}-\mathrm{e}^{-t H_{1}}\right) .
\end{aligned}
$$

Here we have used the definition of the SSF in Remark 5.1, and the last equality follows from Kreun's trace formula (5.1). The semigroup difference in the last line of (2.7) is trace class (cf. Remark 5.1), and possesses a continuous integral kernel. Thus, [Br, Thm. 3.1] justifies the evaluation of the trace by an integral over the diagonal of the kernel so that

$$
\begin{aligned}
\widetilde{\xi}(t) & =\frac{1}{t} \frac{1}{(2 \pi t)^{d / 2}} \int_{\mathbb{R}^{d}} \mathrm{~d} x \mathbb{E}_{x, x}^{0, t}\left[\mathrm{e}^{-\int_{0}^{t} \mathrm{~d} s V_{0}(b(s))}\left(1-\mathrm{e}^{-\int_{0}^{t} \mathrm{~d} s V(b(s))}\right)\right] \\
& =\frac{1}{(2 \pi t)^{d / 2}} \int_{0}^{1} \mathrm{~d} \lambda \int_{\mathbb{R}^{d}} \mathrm{~d} x \int_{0}^{t} \frac{\mathrm{d} s}{t} \mathbb{E}_{x, x}^{0, t}\left[V(b(s)) \mathcal{U}_{t}(b)\right]
\end{aligned}
$$

where we have used (2.2) and Fubini's Theorem. We infer that

$$
\widetilde{\xi}(t)=\lim _{L \rightarrow \infty} \xi_{L}(t)
$$

for every $t>0$. In particular, the limit (2.5) is seen to be finite (as was used earlier). So the claim (1.6) follows from [F, Thm. XIII.1.2a] for $f=\chi_{I}$, with $I \subset \mathbb{R}$ being any (finite) interval. The statement (1.7) is then a consequence of Lebesgue's Differentiation Theorem. Since the supports of all measures involved are uniformly bounded from below, convergence in (1.6) also holds if $f=\chi_{]-\infty, E]}$ for $E \in \mathbb{R}$, that is, for the corresponding distribution functions. But this, in turn, implies that (1.6) holds for all $f \in C_{c}(\mathbb{R})$; see e.g. [B] or HupLMW, Prop 4.3]. The proof of Theorem 1.4 is now complete. 


\section{Proof of Theorem 1.3 on the Cesìro sums}

Since the SSF $\xi_{L}$ is non-negative for every $L>0$, Theorem 1.4 implies that for every sequence $\left(L_{n}\right)_{n \in \mathbb{N}}$ of lengths, which is divergent to $+\infty$, we have

$$
\sup _{n \in \mathbb{N}} \int_{-j}^{j} \mathrm{~d} E \xi_{L_{n}}(E)<\infty
$$

for every fixed $j \in \mathbb{N}$; that is, the sequence is norm bounded in $\mathrm{L}^{1}([-j, j])$. Interpreting $\left(\xi_{L_{n}}\right)_{n \in \mathbb{N}}$ as a sequence of uniformly distributed random variables on $[-j, j]$, Komlós' Theorem [Ko, Thm. 1a] ensures the existence of a subsequence $\left(L_{n_{\nu}^{(j)}}\right)_{\nu \in \mathbb{N}}$ of lengths and of a function $\psi_{j} \in \mathrm{L}^{1}([-j, j])$ such that for every further subsequence $\widetilde{L}_{k}^{(j)}:=L_{n_{\nu_{k}}^{(j)}}, k \in \mathbb{N}$, the Cesàro limit

$$
\lim _{K \rightarrow \infty} \frac{1}{K} \sum_{k=1}^{K} \xi_{\widetilde{L}_{k}^{(j)}}(E)=\psi_{j}(E)
$$

exists for Lebesgue-a.e. $E \in[-j, j]$. Here we can assume without restriction that $\left(n_{\nu}^{(j+1)}\right)_{\nu \in \mathbb{N}}$ is a subsequence of $\left(n_{\nu}^{(j)}\right)_{\nu \in \mathbb{N}}$ for every $j \in \mathbb{N}$. Below we show that

$$
\psi_{j} \leqslant \xi
$$

holds Lebesgue-almost everywhere on $[-j, j]$ for every $j \in \mathbb{N}$. Therefore, given any

subsequence $\widetilde{L}_{k}:=L_{n_{\nu_{k}}^{\left(\nu_{k}\right)}}, k \in \mathbb{N}$, of the sequence $\left(L_{n_{\nu}^{(\nu)}}\right)_{\nu \in \mathbb{N}}$, we get the asserted inequality

$$
\lim _{K \rightarrow \infty} \frac{1}{K} \sum_{k=1}^{K} \xi_{\widetilde{L}_{k}}(E) \leqslant \xi(E)
$$

for Lebesgue-a.e. $E \in \mathbb{R}$.

It remains to establish (3.3) for all $j \in \mathbb{N}$. So fix $j \in \mathbb{N}$ and let $f \in C([-j, j])$ be arbitrary, subject to $f \geqslant 0$ and $f(-j)=0=f(j)$. Then, the trivial extension $F$ of $f$ to $\mathbb{R}$ belongs to the positive cone of $C_{c}(\mathbb{R})$. We conclude from (3.2) and Fatou's Lemma that

$$
\begin{aligned}
\int_{-j}^{j} \mathrm{~d} E f(E) \psi_{j}(E) & =\int_{\mathbb{R}} \mathrm{d} E F(E)\left(\lim _{K \rightarrow \infty} \frac{1}{K} \sum_{k=1}^{K} \xi_{\widetilde{L}_{k}}(E)\right) \\
& \leqslant \int_{\mathbb{R}} \mathrm{d} E F(E) \xi(E)+\liminf _{K \rightarrow \infty} \frac{1}{K} \sum_{k=1}^{K} \mathcal{I}(k),
\end{aligned}
$$

where $\mathcal{I}(k):=\int_{\mathbb{R}} \mathrm{d} E F(E)\left[\xi_{\widetilde{L}_{k}}(E)-\xi(E)\right]$. Now, the vague convergence of Theorem 1.4 guarantees that for every $\varepsilon>0$ there exists $k_{\varepsilon} \in \mathbb{N}$ such that for all $k \geqslant k_{\varepsilon}$ we have $|\mathcal{I}(k)| \leqslant \varepsilon$. This implies

$$
\left|\liminf _{K \rightarrow \infty} \frac{1}{K} \sum_{k=1}^{K} \mathcal{I}(k)\right|=\left|\liminf _{K \rightarrow \infty} \frac{1}{K} \sum_{k=k_{\varepsilon}}^{K} \mathcal{I}(k)\right| \leqslant \varepsilon .
$$

Since $\varepsilon>0$ was arbitrary, we deduce

$$
\int_{-j}^{j} \mathrm{~d} E f(E)\left[\xi(E)-\psi_{j}(E)\right] \geqslant 0
$$


for every non-negative $f \in C([-j, j])$ that vanishes at the boundary points. But this yields (3.3).

\section{Proof of the Birman-Soloymak formula, Theorem 1.6}

In this section we present a simple Feynman-Kac based proof of the BirmanSolomyak formula (1.9). Let us write $\mu(B)$ for the right-hand side of (1.9), which defines a Borel measure $\mu$ on $\mathbb{R}$. We deduce from the spectral theorem and monotone convergence that for every $t>0$ its two-sided Laplace transform $\widetilde{\mu}(t):=$ $\int_{\mathbb{R}} \mathrm{d} \mu(E) \mathrm{e}^{-t E}$ is given by

$$
\begin{aligned}
\widetilde{\mu}(t) & =\int_{0}^{1} \mathrm{~d} \lambda \operatorname{tr}\left[V^{1 / 2} \mathrm{e}^{-t H_{\lambda}} V^{1 / 2}\right]=\int_{0}^{1} \mathrm{~d} \lambda \int_{\mathbb{R}^{d}} \mathrm{~d} x V(x) \mathrm{e}^{-t H_{\lambda}}(x, x) \\
& =\int_{0}^{1} \mathrm{~d} \lambda \int_{\mathbb{R}^{d}} \mathrm{~d} x V(x) \frac{\mathbb{E}_{x, x}^{0, t}\left[\mathcal{U}_{t}(b)\right]}{(2 \pi t)^{d / 2}} .
\end{aligned}
$$

Finiteness of $\widetilde{\mu}(t)$ for $t>0$ and the second equality in (4.1) hold because of $\mathrm{Br}$, Cor. 4.4], the continuity of the integral kernel of the semigroup [S2, Thm. B.7.1(d)] and since $V \in \mathcal{K}\left(\mathbb{R}^{d}\right) \subseteq \mathrm{L}_{\text {unif, loc }}^{1}\left(\mathbb{R}^{d}\right)$ has compact support. The functional $\mathcal{U}_{t}$ was defined in (2.6).

Recall that the probability density $\rho_{x, x}^{0, t}(s ; y)$, where the paths of the Brownian bridge satisfy $b(s)=y \in \mathbb{R}^{d}$ for some $\left.s \in\right] 0, t[$, is given by

$$
\rho_{x, x}^{0, t}(s ; y)=(2 \pi t)^{d / 2} \frac{\mathrm{e}^{-(x-y)^{2} /(2 s)}}{(2 \pi s)^{d / 2}} \frac{\mathrm{e}^{-(x-y)^{2} /[2(t-s)]}}{[2 \pi(t-s)]^{d / 2}} .
$$

The Markov property then amounts to the identity

$$
\begin{aligned}
\mathbb{E}_{x, x}^{0, t}\left[\mathcal{U}_{t}(b)\right] & =\int_{\mathbb{R}^{d}} \mathrm{~d} y \rho_{x, x}^{0, t}(t-s ; y) \mathbb{E}_{x, x}^{0, t}\left[\mathcal{U}_{t}(b) \mid b(t-s)=y\right] \\
& =\int_{\mathbb{R}^{d}} \mathrm{~d} y \rho_{x, x}^{0, t}(t-s ; y) \mathbb{E}_{x, y}^{0, t-s}\left[\mathcal{U}_{t-s}(b)\right] \mathbb{E}_{y, x}^{0, s}\left[\mathcal{U}_{s}(b)\right]
\end{aligned}
$$

for every $s \in] 0, t[$. Hence,

$$
\begin{array}{rl}
\int_{\mathbb{R}^{d}} \mathrm{~d} & x V(x) \mathbb{E}_{x, x}^{0, t}\left[\mathcal{U}_{t}(b)\right] \\
\quad= & \int_{0}^{t} \frac{\mathrm{d} s}{t} \int_{\mathbb{R}^{d}} \mathrm{~d} y \int_{\mathbb{R}^{d}} \mathrm{~d} x \rho_{x, x}^{0, t}(t-s ; y) \mathbb{E}_{y, x}^{0, s}\left[V(b(s)) \mathcal{U}_{s}(b)\right] \mathbb{E}_{x, y}^{0, t-s}\left[\mathcal{U}_{t-s}(b)\right] \\
\quad= & \int_{0}^{t} \frac{\mathrm{d} s}{t} \int_{\mathbb{R}^{d}} \mathrm{~d} y \mathbb{E}_{y, y}^{0, t}\left[V(b(s)) \mathcal{U}_{t}(b)\right]
\end{array}
$$

where the second equality relies on $\rho_{x, x}^{0, t}(t-s ; y)=\rho_{y, y}^{0, t}(s ; x)$ and, again, the Markov property. A comparison of (4.1) and (4.4) with (2.8) reveals that $\widetilde{\mu}(t)=\widetilde{\xi}(t)$ for all $t>0$. Hence, (1.9) follows from [F, Thm. XIII.1.1a].

\section{Appendix: Basics about the SSF}

For the convenience of the reader, we collect some facts related to the SSF in this appendix; see e.g. $[\mathrm{Y}]$. First, we are concerned with its definition in a more general setting. If $A_{0}, A_{1}$ are self-adjoint operators on a Hilbert space $\mathcal{H}$ and if $A_{1}-A_{0}$ is trace class, then $\left[\mathrm{Y}\right.$, Thm. 8.3.3 and following remarks] $f\left(A_{1}\right)-f\left(A_{0}\right)$ is 
trace class for every $f \in C_{c}^{\infty}(\mathbb{R})$ and the SSF $\xi \equiv \xi\left(\cdot ; A_{1}, A_{0}\right)$ of the pair $\left(A_{1}, A_{0}\right)$ is uniquely defined up to an additive constant by Krel̆n's trace formula

$$
\operatorname{tr}\left[f\left(A_{1}\right)-f\left(A_{0}\right)\right]=\int_{\mathbb{R}} \mathrm{d} E f^{\prime}(E) \xi(E) .
$$

The constant can be chosen such that $\xi \in \mathrm{L}^{1}(\mathbb{R})$. In this case, we have the bound $\|\xi\|_{1} \leqslant\left\|A_{1}-A_{0}\right\|_{\text {tr }}$ in terms of the trace norm. We note that the behavior of $f$ outside the union of the spectra of $A_{1}$ and $A_{0}$ is irrelevant for both sides of (5.1) and that (5.1) does also hold for $f$ being the identity.

This definition of the spectral shift function $\xi\left(\cdot ; A_{1}, A_{0}\right)$ can be extended to a pair of self-adjoint operators $\left(A_{1}, A_{0}\right)$ for which it is only known that $\varphi\left(A_{1}\right)-\varphi\left(A_{0}\right)$ is trace class for some function $\varphi \in C^{2}(\Omega)$, where $\Omega$ is a (possibly infinite) interval containing the union of the spectra of $A_{0}$ and $A_{1}$ and where $\varphi$ is bounded and strictly monotone on $\Omega$. In this case we set

$$
\xi\left(E ; A_{1}, A_{0}\right):=\operatorname{sign}\left(\varphi^{\prime}(E)\right) \xi\left(\varphi(E) ; \varphi\left(A_{1}\right), \varphi\left(A_{0}\right)\right)
$$

for all $E \in \Omega$. The SSF on the right-hand side of (5.2) is determined by (5.1), and it is independent of the choice of $\varphi$ within the allowed class of functions (invariance principle) [Y, Sect. 8.11]. Moreover, we have the estimate

$$
\int_{\Omega} \mathrm{d} E\left|\xi\left(E ; A_{1}, A_{0}\right)\right|\left|\varphi^{\prime}(E)\right| \leqslant\left\|\varphi\left(A_{1}\right)-\varphi\left(A_{0}\right)\right\|_{\mathrm{tr}} .
$$

Now we return to the situation of Schrödinger operators as in the main text.

Remark 5.1. Let $d \in \mathbb{N}$ and assume (因). Then, $\mathrm{e}^{-t H_{0}}-\mathrm{e}^{-t H_{1}}$ is trace class for every $t>0$ (see e.g. HuKNSV, Remark after Thm. 1]), and the SSF for the pair $\left(H_{1}, H_{0}\right)$ is defined by (5.2) with $\varphi(E):=\mathrm{e}^{-t E}$ for $E \in \mathbb{R}$. It follows that $\xi\left(\cdot, H_{1}, H_{0}\right) \in \mathrm{L}_{\mathrm{loc}}^{1}(\mathbb{R})$, the integral corresponding to (5.3), is finite and that its Laplace transform $\widetilde{\xi}(t)$ (see (2.7) ) exists for every $t>0$.

\section{ACKNOWLEDGEMENT}

The authors thank François Germinet for repeated kind hospitality at the Université de Cergy-Pontoise, France.

\section{REFERENCES}

[AS] M. Aizenman, B. Simon, Brownian motion and Harnack inequality for Schrödinger operators, Commun. Pure Appl. Math., 35, 209-273, 1982. MR644024 (84a:35062)

[B] H. Bauer, Measure and integration theory. de Gruyter, Berlin, 2001. MR1897176 (2003a:28001)

[BiS] M. Sh. Birman, M. Z. Solomyak, Remarks on the spectral shift function, J. Soviet Math., 3, 408-419, 1975.

[BiY] M. Sh. Birman, D. R. Yafaev, The spectral shift function. The papers of M. G. Kreı̆n and their further development, St. Petersburg Math. J., 4, 833-870, 1993. MR1202723 (94g:47002)

[Br] C. Brislawn, Kernels of trace class operators, Proc. Amer. Math. Soc., 104, 1181-1190, 1988. MR 929421 (89d:47059)

[CHK1] J.-M. Combes, P. D. Hislop, F. Klopp, An optimal Wegner estimate and its application to the global continuity of the integrated density of states for random Schrödinger operators, Duke Math. J., 140, 469-498, 2007. MR2362242 (2009b:82050)

[CHK2] J.-M. Combes, P. D. Hislop, F. Klopp, Some new estimates on the spectral shift function associated with random Schrödinger operators. In: CRM, Proc. Lecture Notes, vol. 42, Probability and mathematical physics, pp. 85-95, Amer. Math. Soc., Providence, RI, 2007. MR2352262(2009b:47063) 
$[\mathrm{CHN}]$ J. M. Combes, P. D. Hislop, S. Nakamura, The $\mathrm{L}^{p}$-theory of the spectral shift function, the Wegner estimate, and the integrated density of states for some random operators, Commun. Math. Phys., 218, 113-130, 2001. MR1824200(2002e:82034)

[F] W. Feller, An introduction to probability theory and its applications. Vol. II, 2nd ed., Wiley, New York, 1971. MR0270403 (42:5292)

[GKS] R. Geisler, V. Kostrykin, R. Schrader, Concavity properties of Krĕn's spectral shift function, Rev. Math. Phys., 7, 161-181, 1995. MR1317338 (96a:47024)

[HM] P. D. Hislop, P. Müller, A lower bound for the density of states of the lattice Anderson model, Proc. Amer. Math. Soc., 136, 2887-2893, 2008. MR2399055 (2009d:82070)

[HuKNSV] D. Hundertmark, R. Killip, S. Nakamura, P. Stollmann, I. Veselić, Bounds on the spectral shift function and the density of states, Commun. Math. Phys., 262, 489503, 2006. MR 2200269 (2006m:81103)

[HuS] D. Hundertmark, B. Simon, An optimal L ${ }^{p}$-bound on the Krein spectral shift function, J. Anal. Math., 87, 199-208, 2002. MR1945282 (2004d:47032)

[HupLMW] T. Hupfer, H. Leschke, P. Müller, S. Warzel, Existence and uniqueness of the integrated density of states for Schrödinger operators with magnetic fields and unbounded random potentials, Rev. Math. Phys., 13, 1547-1581, 2001. MR1869817(2003j:81059)

[K1] W. Kirsch, Small perturbations and the eigenvalues of the Laplacian on large bounded domains, Proc. Amer. Math. Soc., 101, 509-512, 1987. MR908658 (88k:35152)

[K2] W. Kirsch, The stability of the density of states of Schrödinger operators under very small perturbations, Int. Eqns. Op. Th., 12, 383-391, 1989. MR998279 (90f:47071)

[Ko] J. Komlós, A generalization of a problem of Steinhaus, Acta Math. Hungar., 18, 217-229, 1967. MR0210177 (35:1071)

[KosS] V. Kostrykin, R. Schrader, The density of states and the spectral shift density of random Schrödinger operators, Rev. Math. Phys., 12, 807-847, 2000. MR.1770547 (2001d:47056)

[S1] B. Simon, Functional integration and quantum physics, Academic Press, New York, 1979. MR.544188 (84m:81066)

[S2] B. Simon, Schrödinger semigroups, Bull. Amer. Math. Soc. (N.S.), 7, 447-526, 1982. Erratum: ibid., 11, 426, 1984. MR670130 (86b:81001a) MR0752806 (86b:81001b)

[S3] B. Simon, Spectral averaging and the Krein spectral shift, Proc. Amer. Math. Soc., 126, 1409-1413, 1998. MR1443857 (98j:47030)

[So] A. V. Sobolev, Efficient bounds for the spectral shift function, Ann. Inst. Henri Poincaré, 58, 55-83, 1993. MR1208792 (94c:47018)

[Y] D. R. Yafaev, Mathematical scattering theory. General theory, Amer. Math. Soc., Providence, RI, 1992. MR1180965 (94f:47012)

Department of Mathematics, University of Kentucky, Lexington, Kentucky 405060027

E-mail address: hislop@ms.uky.edu

Mathematisches Institut, Ludwig-Maximilians-Universität, Theresienstrasse 39 , 80333 München, Germany

E-mail address: mueller@lmu.de 